\title{
ON THE EXTENSION OF A TRANSFORMATION
}

\author{
EARL J. MICKLE
}

0 . Introduction. In a problem on surface area the writer and Helsel $^{1}$ were confronted with the following question. Can a Lipschitzian transformation from a set in a Euclidean three-space into a Euclidean three-space be extended to a Lipschitzian transformation defined on the whole space? The affirmative answer to this question has been given by Kirszbraun. ${ }^{2}$ In fact, Kirszbraun shows this result for any Euclidean spaces (see also Valentine). ${ }^{3}$ In studying these papers the writer noted that a more general extension problem could be formulated and a different method of proof to the problem could be obtained. To formulate the more general problem we first give some definitions.

Let $M$ be a metric space, the distance between two points $p_{1}, p_{2} \in M$ being denoted by $p_{1} p_{2}$. Let $P(M)$ be the class of real-valued continuous functions $g(t), 0 \leqq t<\infty$, which satisfy the conditions: (a) $g(0)=0$, (b) $g(t)>0$ for $t>0$, (c) for any finite number of points $p_{0}, p_{1}, \cdots, p_{m}$ in $M$ the real quadratic form $\sum_{i, j=1}^{m}\left[g\left(p_{0} p_{i}\right)^{2}+g\left(p_{0} p_{j}\right)^{2}\right.$ $\left.-g\left(p_{i} p_{j}\right)^{2}\right] \xi_{i} \xi_{j}$ is positive. Let $g(t) \in \mathcal{P}(M)$. A transformation $p^{*}=\phi(p)$ from a set $E$ in $M$ into a metric space $M^{*}$ will be said to satisfy the condition $C(g)$ on $E$ if, for every pair of points $p_{1}, p_{2} \in E$, $p_{1}^{*} p_{2}{ }^{*} \leqq g\left(p_{1} p_{2}\right)$, where $p_{i}^{*}=\phi\left(p_{i}\right), i=1,2$. We shall say that $\phi(p)$ can be extended to a set $E^{\prime}, E \subset E^{\prime} \subset M$, preserving the condition $C(g)$ if there exists a transformation $p^{*}=\Phi(p)$ from $E^{\prime}$ into $M^{*}$ which satisfies the condition $C(g)$ on $E^{\prime}$ and is equal to $\phi(p)$ on $E$.

In this paper we prove the following result. Let $M$ be a separable metric space and let $g(t) \in P(M)$. Then any transformation from a set $E$ in $M$ into a Euclidean space which satisfies the condition $C(g)$ on $E$ can be extended to $M$ preserving the condition $C(g)$.

We give two examples to illustrate this result. We shall use the vector notation $x$ to represent a point in a Euclidean $n$-space $E_{n}$, and we shall denote by $\left|x_{1}-x_{2}\right|$ the distance between two points $x_{1}, x_{2}$. Let $x_{0}, x_{1}, \cdots, x_{m}$ be $m+1$ points in $E_{n}$ and let $\xi_{1}, \cdots, \xi_{m}$ be $m$ real numbers. From the relation $\left(x_{i}-x_{j}\right)^{2}=\left(x_{0}-x_{i}\right)^{2}+\left(x_{0}-x_{j}\right)^{2}$ $-2\left(x_{0}-x_{i}\right)\left(x_{0}-x_{j}\right)$, the square of the vector $x=L \xi_{1}\left(x_{0}-x_{1}\right)+\cdots$

\footnotetext{
Received by the editors November 26, 1947, and, in revised form, February 4, 1948.

${ }^{1}$ Helsel and Mickle, Bull. Amer. Math. Soc. vol. 54 (1948) pp. 235-238.

2 Kirszbraun, Fund. Math. vol. 22 (1934) pp. 77-108.

${ }^{3}$ Valentine, Bull Amer. Math. Soc. vol. 49 (1943) pp. 100-108.
} 
$+L \xi_{m}\left(x_{0}-x_{m}\right), L>0$, is given by

$$
\begin{aligned}
x^{2} & =L^{2} \sum_{i, j=1}^{m}\left(x_{0}-x_{i}\right)\left(x_{0}-x_{j}\right) \xi_{i} \xi_{j} \\
& =\frac{L^{2}}{2} \sum_{i, j=1}^{m}\left[\left|x_{0}-x_{i}\right|^{2}+\left|x_{0}-x_{j}\right|^{2}-\left|x_{i}-x_{j}\right|^{2}\right] \xi_{i} \xi_{j} \geqq 0 .
\end{aligned}
$$

Thus the function $g(t)=L t, L>0$, is in $P\left(E_{n}\right)$ and the results of Kirszbraun follow as a special case of the results of this paper. Schoenberg ${ }^{4}$ has shown that the function $g(t)=L t^{\alpha}, L>0,0<\alpha \leqq 1$, is in $P\left(E_{n}\right)$. Thus a transformation from a set in a Euclidean space into a Euclidean space which satisfies a Lipschitz-Hölder condition $(L>0,0<\alpha \leqq 1)$ can be extended to the whole space preserving this same Lipschitz-Hölder condition.

1. Preliminary remarks. In this section we give some well known concepts and lemmas for a Euclidean $n$-space $E_{n}$. A set is called convex if the line segment joining any two points of the set is in the set. If $E$ is a closed convex set and $x \notin E$, then there is a unique point $x^{*} \in E$ which is closest to $x$. (Since $E$ is closed there is one such point. If there were two, the midpoint of the line segment joining them would be in $E$ and closer to $x$ than either of them.) For a finite set of points $x_{1}, \cdots, x_{m}$, we denote by $V\left(x_{1}, \cdots, x_{m}\right)$ the smallest convex set containing them. $V\left(x_{1}, \cdots, x_{m}\right)$ is a closed set consisting of those points given by the relation $x=c_{1} x_{1}+\cdots+c_{m} x_{m}$, where the $c_{i}$ 's are non-negative and $c_{1}+\cdots+c_{m}=1$.

LeMma 1.1. Let $E$ be a closed convex set, $x_{0}$ a point not in $E, x_{0}^{*}$ the unique point of $E$ closest to $x_{0}$, and $y=t x_{0}+(1-t) x_{0}^{*}, 0 \leqq t<1$. Then $|y-x|<\left|x_{0}-x\right|$ for every point $x \in E$.

Proof. Since $|y-x| \leqq t\left|x_{0}-x\right|+(1-t)\left|x_{0}^{*}-x\right|, t \neq 1$, it is sufficient to prove that $\left|x_{0}{ }^{*}-x\right|<\left|x_{0}-x\right|$ for all $x \in E$. Assume there is a point $x_{1} \in E$ for which $\left|x_{0}^{*}-x_{1}\right| \geqq\left|x_{0}-x_{1}\right|$. Then the numbers $a=\left|x_{0}-x_{0}^{*}\right|, b=\left|x_{0}-x_{1}\right|, c=\left|x_{0}^{*}-x_{1}\right|$ satisfy the inequalities $a<b$ $\leqq c$ and the number $t^{*}=\left(a^{2}+c^{2}-b^{2}\right) / 2 c^{2}$ satisfies the inequalities $0<t^{*}<1 / 2$. Thus the point $x=t^{*} x_{1}+\left(1-t^{*}\right) x_{0}^{*}$ is in $E$ and $\left|x_{0}-x\right|^{2}$ $=\left|x_{0}-x_{0}^{*}\right|^{2}-t^{* 2} c^{2}<a^{2}$, contradicting the assumption that $x_{0}^{*}$ is the point of $E$ closest to $x_{0}$.

LEMMA 1.2. Let $\Sigma$ be a set of closed spheres in $E_{n}$ such that there is no point in common to all of them. Then there is a finite set of spheres in $\Sigma$

${ }^{4}$ Schoenberg, Ann. of Math. vol. 38 (1937) pp. 787-793; Amer. J. Math. vol. 67 (1945) pp. 83-93. 
which have no point in common. ${ }^{\mathrm{s}}$

Proof. Let $S_{0}$ be one of the spheres in $\Sigma$. Then the sum of the complements of the remaining spheres cover $S_{0}$. Since this is an open covering of $S_{0}$, there is a finite number of spheres $S_{1}, \cdots, S_{m}$ in $\Sigma$ the sum of whose complements covers $S_{0}$. Hence, $S_{0}, S_{1}, \cdots, S_{m}$ have no point in common.

2. Lemmas. For a given integer $m$, let $a_{1}, \ldots a_{m}$ be a given set of $m$ positive numbers and let $x_{1}, \cdots, x_{m}$ be a given set of (not necessarily distinct) $m$ points in $E_{n}$. For each point $x$ let

$$
f(x)=\max \left(\left|x-x_{i}\right| / a_{i}\right), \quad i=1, \cdots, m .
$$

$f(x)$ is obviously a continuous function of $x$ and there exists a point $x_{0}$ for which $f\left(x_{0}\right)=\min f(x)$. We have the following result concerning the location of a point $x_{0}$ at which $f(x)$ assumes a minimum value.

LEMMA 2.1. Let $x_{0}$ be a point such that $f\left(x_{0}\right)=\min f(x)$ and let $x_{m_{1}}, \cdots x_{m_{k}}$ be all the points in the set of points $x_{1}, \cdots, x_{m}$ for which the equality

$$
f\left(x_{0}\right)=\left|x_{0}-x_{i}\right| / a_{i}
$$

holds. Then $x_{0} \in V\left(x_{m_{1}}, \cdots, x_{m_{k}}\right)$.

Proof. Assume $x_{0} \notin V\left(x_{m_{1}}, \cdots, x_{m_{k}}\right)$. Let $x_{0}{ }^{*}$ be the unique point of $V\left(x_{m_{1}}, \cdots, x_{m_{k}}\right)$ closest to $x_{0}$. For each integer $j$ let $y_{j}=t_{j} x_{0}$ $+\left(1-t_{j}\right) x_{0}{ }^{*}, 0 \leqq t_{j}<1, t_{j} \rightarrow 1$, for $j \rightarrow \infty$. By Lemma 1.1, $\left|y_{j}-x\right|$ $<\left|x_{0}-x\right|$ for every point $x \in V\left(x_{m_{1}}, \cdots, x_{m_{k}}\right)$. Thus, since $f\left(x_{0}\right)$ $\leqq f\left(y_{j}\right), f\left(y_{j}\right)=\left|y_{j}-x_{i}\right| / a_{i}, 1 \leqq i \leqq m$, for some $x_{i} \notin V\left(x_{m_{1}}, \cdots, x_{m_{k}}\right)$. There are an infinite number of the $x_{i}$ 's corresponding to the points $y_{j}$ which are the same and we can assume without loss of generality that all of them are the same point $x_{m_{k+1}}, 1 \leqq m_{k+1} \leqq m$. Since $f\left(y_{j}\right)$ $\rightarrow f\left(x_{0}\right)$ for $j \rightarrow \infty, f\left(x_{0}\right)=\lim \left|y_{j}-x_{m_{k+1}}\right| / a_{m_{k+1}}=\left|x_{0}-x_{m_{k+1}}\right| / a_{m_{k+1}}$, $x_{m_{k+1}} \notin V\left(x_{m_{1}}, \cdots, x_{m_{k}}\right)$. Thus (2.2) holds for $i=m_{k+1}$. This contradicts the fact that (2.2) holds only for the points $x_{m_{1}}, \cdots, x_{m_{k}}$. Hence $x_{0} \in V\left(x_{m_{1}}, \cdots, x_{m_{k}}\right)$.

We now prove the fundamental lemma of the paper.

Lemma 2.2. Let $M$ be a metric space, let $g(t) \in \mathscr{P}(M)$ and let $p_{1}, \cdots, p_{m}$ be $m$ distinct points and $x_{1}, \cdots, x_{m}$ be $m$ points in $M$ and $E_{n}$ respectively for which the inequalities $\left|x_{i}-x_{j}\right| \leqq g\left(p_{i} p_{j}\right)$, $i, j=1, \cdots, m$, hold. Then, for any point $p_{0} \in M$, there exists a point

${ }^{5}$ We use the term closed sphere to mean the set of points $x$ which satisfy the inequality $\left|x-x_{0}\right| \leqq r$ for fixed $x_{0}$ and $r>0$. 
$x_{0} \in E_{n}$ for which the inequalities $\left|x_{0}-x_{i}\right| \leqq g\left(p_{0} p_{i}\right), i=1, \cdots, m$, hold.

Proof. If $p_{0}=p_{i}, 1 \leqq i \leqq m$, let $x_{0}=x_{i}$. Assume $p_{0} \neq p_{1}, \cdots, p_{m}$. Set $a_{i}=g\left(p_{0} p_{i}\right), i=1, \cdots, m$. Since $g(t) \in \mathbb{P}(M)$, each $a_{i}>0$. If $x_{0}$ is a point for which $f\left(x_{0}\right)=\min f(x)$ (see (2.1)), we assert that $\lambda=f\left(x_{0}\right)$ $\leqq 1$. That is to say, $\left|x_{0}-x_{i}\right| \leqq g\left(p_{0} p_{i}\right), i=1, \cdots, m$. If $\lambda=0$, then $\lambda \leqq 1$. Assume $\lambda>0$. Set $a_{i j}=g\left(p_{i} p_{j}\right), b_{i j}=\left|x_{i}-x_{j}\right|$ and $b_{i}=\left|x_{0}-x_{i}\right|$, $i, j=1, \cdots, m$. By renumbering if necessary, let $x_{1}, \cdots, x_{k}$ be the points for which the equality $f\left(x_{0}\right)=b_{i} / a_{i}=\lambda$ holds. By Lemma 2.1, $x_{0} \in V\left(x_{1}, \cdots, x_{k}\right)$. Thus we have non-negative numbers $c_{1}, \cdots, c_{k}$, $c_{3}+\cdots+c_{k}=1$ such that $x_{0}=c_{1} x_{1}+\cdots+c_{k} x_{k}$ or $c_{1}\left(x_{0}-x_{1}\right)+\cdots$ $+c_{k}\left(x_{0}-x_{k}\right)=0$. By squaring this expression and using the relation $\left(x_{i}-x_{j}\right)^{2}=\left(x_{0}-x_{i}\right)^{2}+\left(x_{0}-x_{j}\right)^{2}-2\left(x_{0}-x_{i}\right)\left(x_{0}-x_{j}\right)$ we obtain

$$
\sum_{i, j=1}^{k}\left(x_{0}-x_{i}\right)\left(x_{0}-x_{j}\right) c_{i} c_{j}=\frac{1}{2} \sum_{i, j=1}^{k}\left(b_{i}^{2}+b_{j}^{2}-b_{i j}^{2}\right) c_{i} c_{j}=0 .
$$

Since $\lambda>0, x_{0} \neq x_{1}, \cdots, x_{k}$, and hence at least two of the $c_{i}$ 's are different from zero. Since $g(t) \in \mathcal{P}(M)$, the quadratic form $\sum_{i, j=1}^{k}\left(a_{i}^{2}+a_{j}^{2}\right.$ $\left.-a_{i j}^{2}\right) \xi_{i} \xi_{j}$ is positive. Setting $\xi_{i}=\lambda c_{i}, i=1, \cdots, k$, and using the fact that $\lambda a_{i}=b_{i}, i=1, \cdots, k$, we obtain

4) $\frac{1}{2} \sum_{i, j=1}^{k}\left(a_{i}^{2}+a_{j}^{2}-a_{i j}^{2}\right) \lambda^{2} c_{i} c_{j}=\frac{1}{2} \sum_{i, j=1}^{k}\left(b_{i}^{2}+b_{j}^{2}-\lambda^{2} a_{i j}^{2}\right) c_{i} c_{j} \geqq 0$.

Subtracting (2.3) from (2.4) gives

$$
\frac{1}{2} \sum_{i, j=1}^{k}\left(b_{i j}^{2}-\lambda^{2} a_{i j}^{2}\right) c_{i} c_{j} \geqq 0 .
$$

Since $a_{i j}=b_{i j}=0$ for $i=j$, the $c_{i}$ 's are non-negative and at least two of the $c_{i}$ 's are different from zero, it follows from (2.5) that $b_{i j}^{2}-\lambda^{2} a_{i j}^{2} \geqq 0$ for some pair of integers $i, j$ with $i \neq j$. For this pair of integers $1 \leqq i, j \leqq k, i \neq j$, we have $g\left(p_{i} p_{j}\right)^{2} \geqq\left|x_{i}-x_{j}\right|^{2}=b_{i j}^{2} \geqq \lambda^{2} a_{i j}^{2}=\lambda^{2} g\left(p_{i} p_{j}\right)^{2}$. Hence $\lambda \leqq 1$. Thus a point $x_{0}$ at which $f(x)$ assumes a minimum satisfies the conditions of the lemma.

LEMmA 2.3. Let $M$ be a metric space, let $g(t) \in P(M)$, let $x=\phi(p)$ be a transformation from a set $E$ in $M$ into $E_{n}$ and let $p_{0}$ be any point in $M$. Then, if $\phi(p)$ satisfies the condition $C(g)$ on $E, \phi(p)$ can be extended to $E+p_{0}$ preserving the condition $C(g)$.

Proof. If $p_{0} \in E$ the extension is immediate. Assume $p_{0} \notin E$. For each $p \in E$, let $S_{p}$ be the set of points $x \in E_{n}$ which satisfy the in- 
equality $|x-\phi(p)| \leqq g\left(p_{0} p\right)$. Since $g(t) \in \mathcal{P}(M)$, each $S_{p}$ is a closed sphere in $E_{n}$. Assume that there is no point in common to all the spheres. By Lemma 1.2 there is a finite number of these spheres which have no point in common. This contradicts Lemma 2.2. Hence, there is at least one point $x_{0}$ in all the spheres $S_{p}, p \in E$. Then $\Phi\left(p_{0}\right)=x_{0}, \Phi(p)=\phi(p), p \in E$, is an extension of $\phi(p)$ to $E+p_{0}$ preserving the condition $C(g)$.

3. The main result. We now state and prove the main result of this paper.

TheOREM. Let $M$ be a separable metric space, let $g(t) \in \mathcal{P}(M)$ and let $x=\phi(p)$ be a transformation from a set $E$ in $M$ into a Euclidean space $E_{n}$. Then if $\phi$ satisfies the condition $C(g)$ on $E, \phi$ can be extended to $M$ preserving the condition $C(\mathrm{~g})$.

Proof. Let $D$ be a finite or denumerable set which is dense in $M$. By Lemma $2.3, \phi(p)$ can be extended to $E$ plus any point of $D$ and by induction to $E+D$ preserving the condition $C(g)$. Let $x=\Phi(p)$, $p \in E+D$ be the extended transformation. Since $E_{n}$ is complete and $g(t) \in \mathcal{P}(M)$, a convergent sequence of points $p_{m} \in E+D, \quad m=1$, $2, \cdots$, determines a convergent sequence of points $\Phi\left(p_{m}\right)$ in $E_{n}$. Since $E+D$ is dense in $M, \Phi(p)$ can be extended to $M$ preserving the condition $C(g)$ in one and only one way.

4. Additional remarks. The writer is indebted to the referee for pointing out the following facts. Any finite set of points in a unitary space is isometrically equivalent to a set of points in some Euclidean space. Hence Lemma 2.2 is valid in any unitary space. Lemma 1.2 is valid in any complete unitary space (see Murray ${ }^{6}$ for the case where the space is separable and Alaoglu ${ }^{7}$ for the general case). Hence Lemma 2.3 is valid if $E_{n}$ is replaced by a complete unitary space. Then the theorem in $\S 3$ with $E_{n}$ replaced by a complete unitary space $U$ and with $M$ not assumed to be separable follows from Lemma 2.3 (with $E_{n}$ replaced by $U$ ) by applying Zorn's lemma or transfinite induction.

\section{The Ohio State University}

${ }^{6} \mathrm{~F}$. J. Murray, Linear transformations in Hilbert space, Princeton University Press, 1941.

${ }^{7}$ Alaoglu, Ann. of Math. vol. 41 (1940) pp. 252-267. 